\title{
Interpretation of Alkyl Diselenide and Selenosulfenate Mass Spectra
}

\author{
Juris Meija, Thomas L. Beck, and Joseph A. Caruso \\ Department of Chemistry, University of Cincinnati, Cincinnati, Ohio, USA
}

\begin{abstract}
The mass spectral fragmentation of aliphatic diselenides and selenosulfenates is analyzed to gain a better understanding of the behavior of these species. The main fragmentation pathways of these species include the fragmentation along the Se-C bond, fragmentation along the Se-Se or Se-S bonds and intra-molecular rearrangements. In general, negative ionization favors the fragmentation along the Se-Se or Se-S bonds while positive ionization leads to stable molecular ions. Density functional theory calculations of bond dissociation energies and molecular orbital analysis was undertaken to explain the observed trends in molecular fragmentation. Besides the analysis of molecular fragmentation, a phenomenon of molecular association in negative electron impact and positive chemical ionization conditions was observed and investigated using a high resolution time-of-flight mass spectrometer. Molecular association that occurs during the ionization of species includes the formation of symmetrical diselenides from asymmetrical selenosulfenates and formation of alkylseleno adducts from the corresponding diselenides. For species which is hard to resolve by mass analysis, such as isobars of $\mathrm{CHSe}, \mathrm{CH}_{2} \mathrm{Se}$, and $\mathrm{CH}_{3} \mathrm{Se}$, the isotope pattern superimposition procedure was applied to define the overlapping clusters. (J Am Soc Mass Spectrom 2004, 15, 1325-1332) (C) 2004 American Society for Mass Spectrometry
\end{abstract}

$\mathrm{I}$ nterest in selenium species has recently increased because of their anti-oxidative and anti-carcinogenic properties. As a result, speciation of selenium metabolites in the environment has been undertaken by many research groups to gain more understanding about bio-transformations and the occurrence of the various selenium compounds. Inductively coupled plasma mass spectrometry (ICP-MS) is usually utilized for efficient detection and screening of the various selenium species at ultra-trace levels; however, the ultimate identification necessitates characterization by molecular mass spectrometry [1].

Volatile selenium species are of interest as the end terminal in Se metabolism, and are emitted by plants as a means of self-detoxification [2]. Additionally, diselenides and selenosulfenates are red.-ox. active species and account for the anti-oxidative action of selenium [3]. Despite the interest in the selenium volatiles, very few reports are devoted to the investigation of mass spectral fragmentation of heavier dichalcogenidesdiselenides (R-SeSe-R) and selenosulfenates (R-SeS-R). Early mass spectrometric studies of organic selenium compounds were undertaken by Rebane [4-6] and recently by Prabhakar et al. [7]. However, diselenides and selenosulfenates were not a subject of interest in either of the studies.
In this study we analyzed the mass spectral fragmentation of methyl-, ethyl- and ethylmethyl- diselenides and selenosulfenates to gain a better understanding of the behavior of these species. In order to explain the fragmentation behavior of diselenides and selenosulfenates, density functional theory calculations were performed to obtain the bond dissociation energies and molecular orbital information.

\section{Experimental}

\section{Reagents and Standards}

All reagents were of analytical grade and were used without any further purification. Dimethyl disulfide was purchased from Fluka (Milwaukee, WI). Dimethyl trisulfide, dimethyl diselenide, diethyl disulfide and heptacosafluorotributylamine were purchased from Sigma-Aldrich (Milwaukee, WI). Diethyl diselenide was purchased from Strem Chemicals (Newburyport, MA). Chloro- and bromo-pentafluorobenzene, and 2,4,6-tris(trifluoromethyl)-1,3,5-triazine were purchased from Lancaster (Pelham, NH). Asymmetric diselenides and selenosulfenates were prepared in the mixture as described elsewhere [8].

\section{Instrumentation}

Published online July 27, 2004
Address reprint requests to Dr. J. A. Caruso, Department of Chemistry, University of Cincinnati, P.O. Box 210172, Cincinnati, OH 45221-0172, USA E-mail: joseph.caruso@uc.edu
A high resolution Micromass GCT ${ }^{\mathrm{TM}}$ orthogonal timeof-flight mass spectrometer (Micromass, Manchester, 
UK) coupled to GC was used for mass spectral characterization of the synthesized selenium species. High mass accuracy and precision is achieved by continuous leaking of a single reference compound directly into the ionization source from the reference inlet. A single fragment of the reference compound is used as an internal reference signal (lock mass). Accurate mass measurements were performed after calibrating the instrument using heptacosafluorotributylamine in EI+, $\mathrm{EI}-, \mathrm{CI}+, \mathrm{CI}-$, and FI+ ionization modes (with no reagent gas in $\mathrm{CI}+$ mode during the calibration). Heptacosafluorotributylamine was used also as a lock mass compound in EI+ ionization mode (218.9856 u), 2,4,6tris-(trifluoromethyl)-1,3,5-triazine in $\mathrm{CI}+$ mode $(286.0027 \mathrm{u})$ and chloro- and bromo- pentafluorobenzene in CI- mode (201.9609 $u$ and $245.9104 \mathrm{u}$, respectively). Instrumental grade isobutane was used as a reagent gas. If the average mass accuracy exceeded 0.001-0.002 $\mathrm{u}$ the instrument was recalibrated. The estimated resolution of the instrument, $m / \Delta m=3700$. Field ionization filaments were obtained from Carbotech (Carbotech, Germany).

An Agilent $6890 \mathrm{~N}$ gas chromatograph was utilized in this work (Agilent Technologies; Palo Alto, CA). Instrumental conditions for the separation of the species are described elsewhere [8].

\section{Computational Details}

All calculations were performed with the Gaussian 98 implementation of Becke's three-parameter hybrid density functional (B3LYP) theory [9]. All geometries for the studied compounds were fully optimized without using symmetry or structural constraints using the Berny optimization and the tight Gaussian 98 convergence criteria [9]. The locally dense triple split valence basis set, $6-311+G(2 d)$, was found to give good results (in terms of result consistency and computational cost) and was used for geometry optimization and normal vibrational mode analysis. Vibrational frequency calculations were performed on each of the optimized structures and no imaginary values were found. The zeropoint energies and the corresponding thermal corrections to the enthalpy and Gibbs free energy at $298.15 \mathrm{~K}$ were obtained by using the harmonic oscillator and rigid rotor approximations and were subsequently added to the total electronic energies to estimate the enthalpy and Gibbs free energy of the species. Population analysis was calculated using Mulliken and natural bond orbital method [10], both implemented in Gaussian 98. As the population analysis is basis-set sensitive, the results of both methods were compared for various basis sets until consistent results were obtained (with 3-21G and 6-31G basis sets).

Isobaric interferences, such as ${ }^{80} \mathrm{Se}$ and $\mathrm{H}_{2}^{78} \mathrm{Se}$ etc. were evaluated using isotope pattern superimposition principle in conjunction with the least square optimization principle, where the isotope pattern is recon-
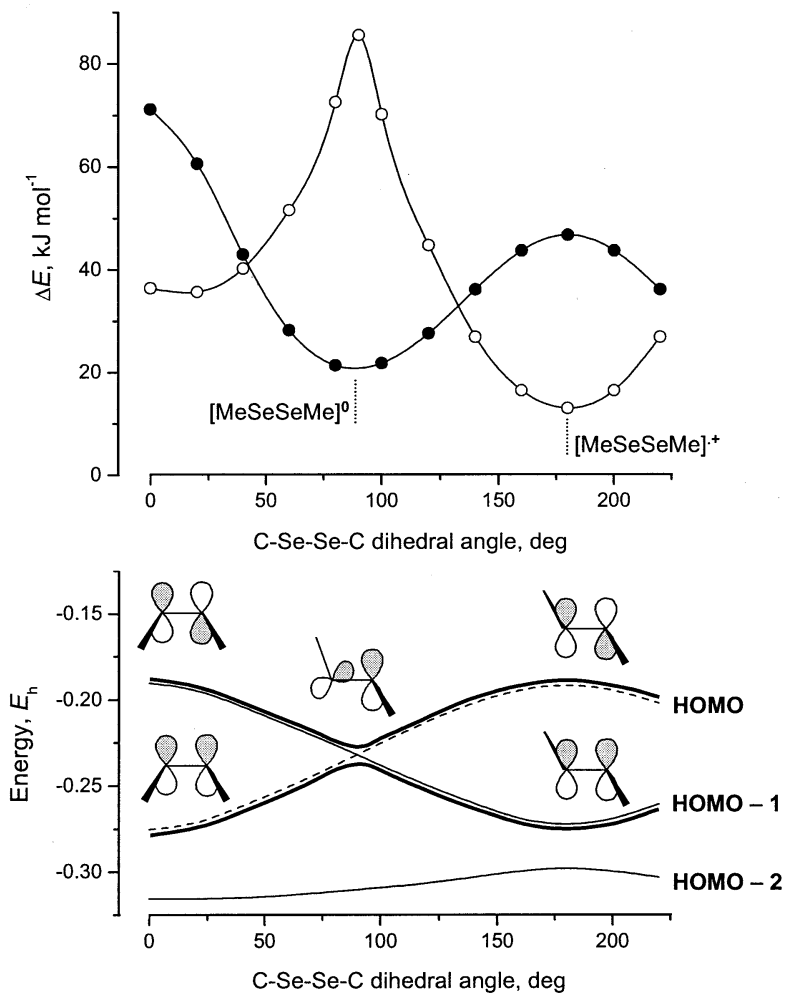

Figure 1. Removal of one electron from diselenides results in planar geometry of the obtained radical cation. Note that the electron is removed from the anti-bonding $\pi-\pi^{*}$ molecular orbital. Calculated using ub3lyp/6-311+G(2d).

structed from its possible contributions using the pattern matching optimization [11].

\section{Results and Discussion}

\section{EI+ Ionization}

In general, alkyl disulfides, selenosulfenates and diselenides give intense molecular ions in EI+ spectra. This can be easily explained by the properties of electronic structure of these species. The highest occupied molecular orbital (HOMO) in these compounds is a set of two perpendicular (non-bonding) lone pairs on chalcogen atoms (Figure 1) and the removal of an electron from diselenides or selenosulfenates (and disulfides) results in planar radical cation where the electron is removed from the anti-bonding $\pi-\pi$ orbital, thus increasing the $\mathrm{Se}-\mathrm{Se}, \mathrm{Se}-\mathrm{S}$ and $\mathrm{Se}-\mathrm{C}, \mathrm{S}-\mathrm{C}$ bond energies (Figure 1 and Table 1). This explains the inherent stability of the positively charged molecular ions of diselenides and selenosulfenates observed in positive ionization.

EI+ fragmentation of dimethyldiselenide starts with the removal of electron from the molecule. From here, further transformations occur according to the three main pathways: (1) Fragmentation along the Se-C bond, (2) fragmentation along the Se-Se bond, and (3) intramolecular rearrangement yielding selenoseleninyl radical cation, $\mathrm{R}_{2} \mathrm{Se}^{-} \mathrm{Se}^{++}$(Scheme $\mathbf{1}$ ). 
Table 1. Calculated bond dissociation energies of dimethyldiselenide

\begin{tabular}{lcc}
\hline Species & $\begin{array}{c}\mathrm{E}(\mathrm{C}-\mathrm{Se}), \\
\mathrm{kJ} \mathrm{mol}^{-1}\end{array}$ & $\begin{array}{r}\mathrm{E}(\mathrm{Se}-\mathrm{Se}), \\
\mathrm{kJ} \mathrm{mol}^{-1}\end{array}$ \\
\hline \hline MeSeSeMe & 198 & $202^{\mathrm{a}}$ \\
MeSeSeMe & 274 & 433 \\
$\mathrm{MeSeSeMe}^{--}$ & 98 & 100 \\
\hline
\end{tabular}

Method of calculation: ub3lyp/6-311+G(2d)

aExperimental value of $192 \pm 12 \mathrm{~kJ} \mathrm{~mol}^{-1}$ is given by Telńoi et al, [15].

Fragmentation along the $\mathrm{Se}-\mathrm{C}$ bond is the main fragmentation pathway in dimethyldiselenide and similar compounds. This seems to occur by (sequential) loss of $\mathrm{CH}_{3}$ radicals leading to the $\mathrm{MeSeSe}^{+}$and $\mathrm{SeSe}^{-+}$(see Figure 2). In positive ionization mode, fragmentation along the Se-Se bond is not highly favorable as the least energy demanding route is the cleavage of $\mathrm{Se}-\mathrm{C}$ bond (see Table 1). Besides that, $\mathrm{MeSe}-\mathrm{Se}^{+}$cations formed during the Se-C bond cleavage can be stabilized by atomic charge dislocation via the resonance equilibria $\left[\mathrm{Me}-\mathrm{Se}-\mathrm{Se}^{+} \leftrightarrow \mathrm{Me}-\mathrm{Se}^{+}=\mathrm{Se}\right]$, which is reflected in the atomic charges on the selenium atom in this species (both Se atoms bear equal positive charge of 0.5). Evidently, cleavage along the Se-Se bond is not greatly favored, as indicated by the low abundance of $\mathrm{CH}_{3} \mathrm{Se}^{+}$ at $m / z=95$ (see Figure 3).

Intra-molecular rearrangement of dimethyldiselenide or dimethylselenosulfenate is evidenced by the formation of a $\mathrm{C}_{2} \mathrm{H}_{5}$ Se cluster as it can logically occur only via the loss of $\mathrm{HSe}$ radical from the dimethylselenoseleninyl radical cation, $\mathrm{Me}_{2} \mathrm{Se}-\mathrm{Se}^{\cdot+}$, yielding $\mathrm{CH}_{2}=\mathrm{Se}-\mathrm{CH}_{3}^{+}(\mathrm{m} / z=108.9556)$. The presence of both $-\mathrm{C}_{2} \mathrm{H}_{5} \mathrm{~S}^{+}$and $\mathrm{C}_{2} \mathrm{H}_{5} \mathrm{Se}^{+}$ions in the EI+ mass spectra of dimethylselenosulfenate suggests that intramolecular methylgroup rearrangement of $\mathrm{MeSeSMe}^{\cdot+}$ yields both -thioseleninyl $\left(\mathrm{Me}_{2} \mathrm{Se}-\mathrm{S}^{\cdot+}\right)$ and selenosulfaninyl $\left(\mathrm{Me}_{2} \mathrm{~S}-\mathrm{Se}^{\cdot+}\right.$ ) ions (Table 2).

This is also in agreement with the total atomic charge distribution in dimethylselenosulfenate radical cation (molecular ion). Atomic charges on sulfur and selenium are close $(+0.3$ and +0.6 respectively), and so are the atomic charges on both carbon atoms $(-1.0)$. In such a case atomic charge-driven methylgroup migration can occur for either of them in MeSeSMe ${ }^{-+}$. Table 2 summarizes the fragments characteristic of the alkylgroup migration that cannot be attributed to the direct fragmentation. Note that the abundance of some these ions approaches up to $10-15 \%$ of the molecular ion.

Rearrangements seen from dimethyldiselenide or selenosulfenate suggest 4-member ring hydrogen transfer. However, species like diethyldiselenide or diethylselenosulfenate, in addition to the loss of $\mathrm{CH}_{3} \mathrm{Se}$ radical show also the loss of HSe radical (Table 2), which is consistent with a 5-member ring hydrogen transfer rearrangement (Scheme 2).

The observed selenoseleninyl rearrangement is disadvantageous since it may be confused with the presence of ethylseleno ion $\left(\mathrm{CH}_{3} \mathrm{CH}_{2} \mathrm{Se}^{+}\right.$versus $\mathrm{CH}_{2} \mathrm{SeCH}_{3}^{+}$), which is the only mass spectrometric (in $\mathrm{EI}+$ ) evidence that the ethyl group is directly attached to the selenium atom. Internal rearrangement is especially disturbing in the case of asymmetrical species, such as MeSeSEt and EtSeSMe, where differences between the structural isomers cannot be conclusively elucidated by inspecting EI+ fragmentation patterns. Additionally, the use of GC retention time information is limited because of the very close structural properties and the lack of pure standards for asymmetrical selenosulfenates.

Relatively low abundance of methylseleno cation, $\mathrm{CH}_{3}^{80} \mathrm{Se}^{+}(\mathrm{m} / \mathrm{z}=95)$ with respect to $\mathrm{m} / z=93$ (usually ascribed to $\mathrm{CH}_{3}^{78} \mathrm{Se}^{+}$) has been a subject of confusion [12]. The fact that $m / z=93$ abundance in EI+ spectra of dimethyldiselenide is of higher abundance than of $\mathrm{m} / \mathrm{z}$ $=95$ cannot be explained only by the signal assignment to $\mathrm{CH}_{3} \mathrm{Se}^{+}$because the abundance of ${ }^{78} \mathrm{Se}$ is about two times lower than that of ${ }^{80} \mathrm{Se}$ (see Figure 3 ). The distorted natural selenium isotope pattern suggests the presence of several isobaric species. Isobars of $\mathrm{CH}_{n} \mathrm{Se}$

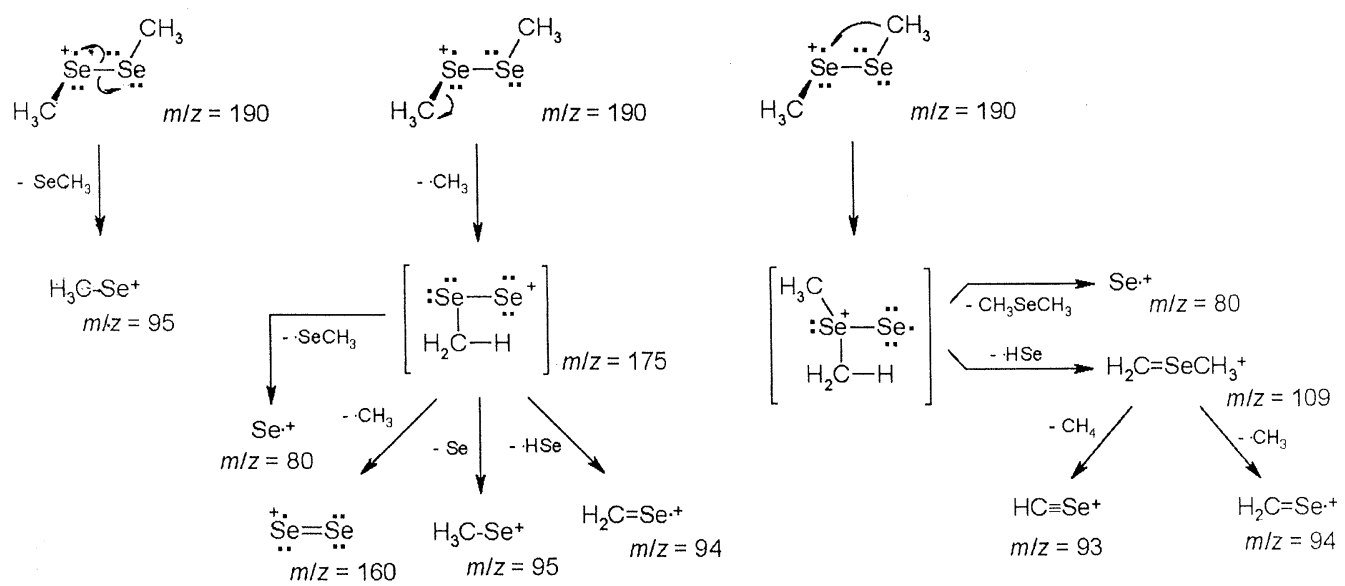

Scheme 1. Mass spectral fragmentation of the dimethyldiselenide in EI+ ionization mode (mass based on $\left.{ }^{80} \mathrm{Se}\right)$. 

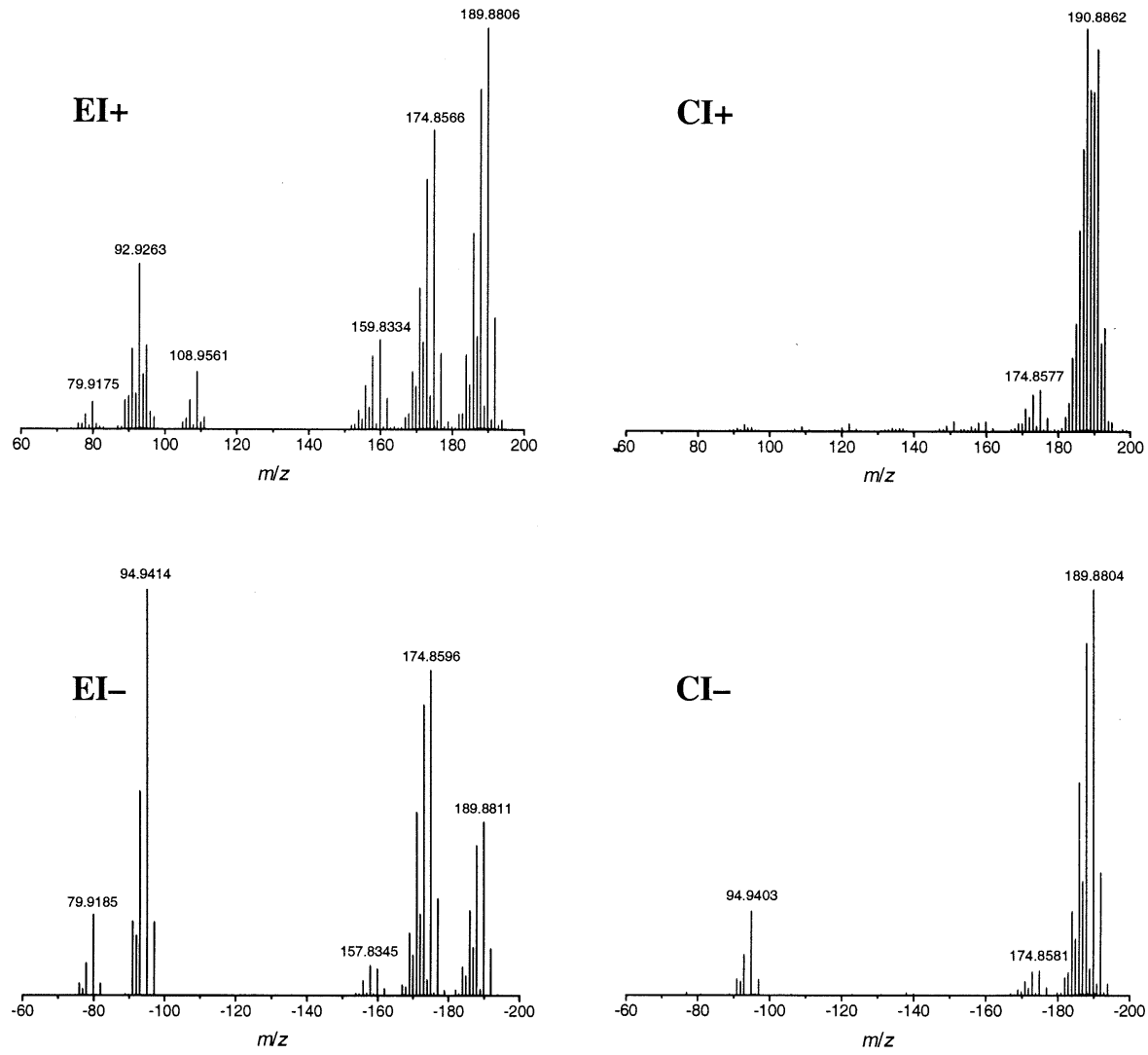

Figure 2. High resolution mass spectra of dimethyldiselenide in $\mathrm{EI}+, \mathrm{EI}-, \mathrm{CI}+$, and $\mathrm{CI}-$ ionization modes.

$\left(\mathrm{CH}^{80}\right.$ Se and $\mathrm{CH}_{3}^{78}$ Se for example) can be resolved only on instruments with high mass resolving capability of $m / \Delta m>10,000$. To elucidate the identity of species that form the isotopic pattern around $\mathrm{m} / \mathrm{z}=89-98$ in the mass spectra of dimethyldiselenide, the cluster observed in EI+ spectra was mathematically recon- structed allowing for contributions of $\mathrm{CH}_{n} \mathrm{Se}$ species where $n=0-4$ [11]. The results show that the $\mathrm{CH}_{n} \mathrm{Se}$ cluster in $\mathrm{CH}_{3} \mathrm{Se}$-containing species (such as MeSeSMe, MeSeSeMe, MeSeSEt etc.) consists of $\mathrm{CHSe}^{+}, \mathrm{CH}_{2} \mathrm{Se}^{-+}$, and $\mathrm{CH}_{3} \mathrm{Se}^{+}$in the amount ratio of about 2:1:1. Note that $\mathrm{CH}_{3} \mathrm{Se}^{+}$contributes only about $25 \%$ to the $\mathrm{CH}_{n} \mathrm{Se}^{+}$

Table 2. Alkylgroup rearrangements in EI+ ionization of dichalcogenides (see Scheme 2)

\begin{tabular}{|c|c|c|c|c|}
\hline Compound & Rearrangement & Fragmentation & Fragment ion (intensity) $^{a}$ & Measured mass (error) ${ }^{\mathrm{b}}$ \\
\hline Me-SeSe-Me & Me-migration & {$[\mathrm{M}-\mathrm{HSe}]$} & $\mathrm{CH}_{3}-\mathrm{Se}=\mathrm{CH}_{2}+(15 \%)$ & $108.9566 \mathrm{u}(+1.0 \mathrm{mu})$ \\
\hline \multirow[t]{2}{*}{$M e^{1}-S e S-M e^{2}$} & $\mathrm{Me}^{2}$-migration & {$[\mathrm{M}-\mathrm{HS}]$} & $\mathrm{CH}_{3}-\mathrm{Se}=\mathrm{CH}_{2}^{+}(2 \%)$ & 108.9559 u $(+0.3 \mathrm{mu})$ \\
\hline & $\mathrm{Me}^{1}$-migration & {$[\mathrm{M}-\mathrm{HSe}]$} & $\mathrm{CH}_{3}-\mathrm{S}=\mathrm{CH}_{2}^{+}(8 \%)$ & $61.0115 \mathrm{u}(+0.3 \mathrm{mu})$ \\
\hline \multirow[t]{2}{*}{ MeSeSe-Et } & $\begin{array}{l}\text { Me-migration } \\
\text { Et-migration }\end{array}$ & {$[\mathrm{M}-\mathrm{HSe}]$} & $\begin{array}{l}\mathrm{CH}_{3} \mathrm{CH}_{2}-\mathrm{Se}=\mathrm{CH}_{2}^{+}(0.4 \%) \\
\mathrm{CH}_{3}-\mathrm{Se}-\mathrm{CH}_{2} \mathrm{CH}_{2}^{+}\end{array}$ & $122.9703 \mathrm{u}(-1.0 \mathrm{mu})$ \\
\hline & $\begin{array}{l}\text { Me-migration } \\
\text { Et-migration }\end{array}$ & {$\left[\mathrm{M}-\mathrm{CH}_{3} \mathrm{Se}\right]$} & $\mathrm{CH}_{3}-\mathrm{Se}=\mathrm{CH}_{2}^{+}(-)$ & Overlaps with $\mathrm{EtSe}^{+}$ \\
\hline \multirow[t]{4}{*}{ Me-SSe-Et } & Et-migration & [M - HSe] & $\mathrm{CH}_{3} \mathrm{CH}_{2}-\mathrm{S}=\mathrm{CH}_{2}^{+}(0.4 \%)$ & $75.0271 \mathrm{u}(+0.3 \mathrm{mu})$ \\
\hline & Et-migration & {$\left[\mathrm{M}-\mathrm{CH}_{3} \mathrm{Se}\right]$} & $\mathrm{CH}_{3}-\mathrm{S}=\mathrm{CH}_{2}{ }^{+}(1 \%)$ & $61.0117 \mathrm{u}(+0.5 \mathrm{mu})$ \\
\hline & Me-migration & {$[\mathrm{M}-\mathrm{HS}]$} & $\mathrm{CH}_{3} \mathrm{CH}_{2}-\mathrm{S}=\mathrm{CH}_{2}{ }^{+}(0.4 \%)$ & $75.0271 \mathrm{u}(+0.3 \mathrm{mu})$ \\
\hline & Me-migration & {$\left[\mathrm{M}-\mathrm{CH}_{3} \mathrm{~S}\right]$} & $\begin{array}{l}\mathrm{CH}_{2}-\mathrm{S}-\mathrm{CH}_{2} \mathrm{CH}_{2}^{+} \\
\mathrm{CH}_{3}-\mathrm{Se}=\mathrm{CH}_{2}^{+}(-)\end{array}$ & Overlaps with EtSe ${ }^{+}$ \\
\hline \multirow[t]{2}{*}{ Et-SeSe-Et } & Et-migration & {$[\mathrm{M}-\mathrm{HSe}]$} & $\mathrm{CH}_{3} \mathrm{CH}_{2}-\mathrm{Se}-\mathrm{CH}_{2} \mathrm{CH}_{2}{ }^{+}(0.6 \%)$ & $136.9892 \mathrm{u}(+2.2 \mathrm{mu})$ \\
\hline & Et-migration & {$\left[\mathrm{M}-\mathrm{CH}_{3} \mathrm{Se}\right]$} & $\mathrm{CH}_{3} \mathrm{CH}_{2}-\mathrm{Se}=\mathrm{CH}_{2}^{+}(0.4 \%)$ & $122.9730 \mathrm{u}(+1.7 \mathrm{mu})$ \\
\hline \multirow{4}{*}{$E t^{1}-S e S-E t^{2}$} & $E t^{1}$-migration & {$[\mathrm{M}-\mathrm{HSe}]$} & $\mathrm{CH}_{3} \mathrm{CH}_{2}-\mathrm{S}-\mathrm{CH}_{2} \mathrm{CH}_{2}^{+}(0.2 \%)$ & 89.0421 u $(-0.4 \mathrm{mu})$ \\
\hline & $E t^{1}$-migration & {$\left[\mathrm{M}-\mathrm{CH}_{3} \mathrm{Se}\right]$} & $\mathrm{CH}_{3} \mathrm{CH}_{2}-\mathrm{S}=\mathrm{CH}_{2}^{+}(0.3 \%)$ & $75.0272 \mathrm{u}(+0.4 \mathrm{mu})$ \\
\hline & $\mathrm{Et}^{2}$-migration & [M - HS] & $\mathrm{CH}_{3} \mathrm{CH}_{2}-\mathrm{Se}-\mathrm{CH}_{2} \mathrm{CH}_{2}^{+}(-)$ & Not detected \\
\hline & $\mathrm{Et}^{2}$-migration & {$\left[\mathrm{M}-\mathrm{CH}_{3} \mathrm{~S}\right]$} & $\mathrm{CH}_{3} \mathrm{CH}_{2}-\mathrm{Se}=\mathrm{CH}_{2}{ }^{+}(-)$ & Not detected \\
\hline
\end{tabular}

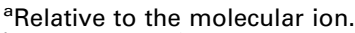

${ }^{b}$ Based on the ${ }^{80} \mathrm{Se}$ ion. Error is given in milli-mass units. 


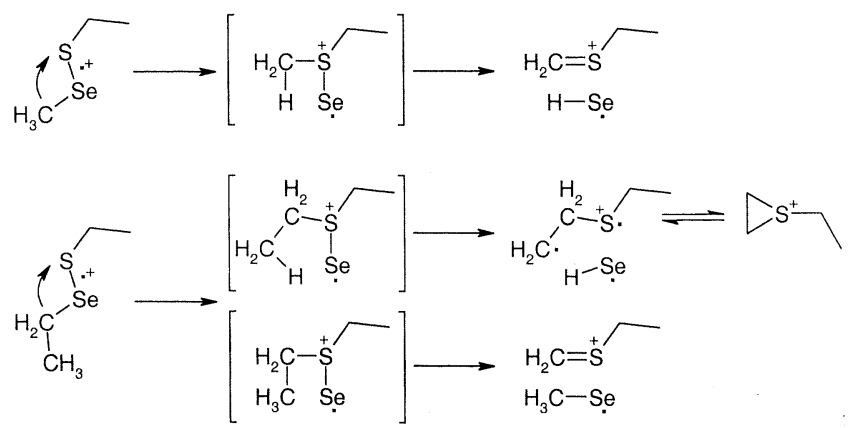

Scheme 2. Alkylgroup rearrangements in EI+ ionization of dichalcogenides.

cluster. $\mathrm{CH}_{n}$ Se cluster is observed also in $\mathrm{CI}+$ ionization while negative ionization renders pure $\mathrm{CH}_{3}$ Se pattern (see Figure 3). This suggests that the formation of $\mathrm{CHSe}^{+}$and $\mathrm{CH}_{2} \mathrm{Se}^{\cdot+}$ is associated with the $\mathrm{Se}-\mathrm{C}$ bond rupture, which is favored in positive ionization (see Scheme 1). Similar to methyl- diselenides and selenosulfenates, $\mathrm{CHS}^{+}$and $\mathrm{CH}_{2} \mathrm{~S}^{++}$also contributes the most in the $\mathrm{CH}_{n} \mathrm{~S}$ pattern of dimethyl disulfide $\mathrm{EI}+$ mass spectra.

Reconstruction of EI+ spectra of ethylselenides has shown that $-\mathrm{CH}_{2} \mathrm{CH}_{3}$ eliminates mainly via the neutral loss of $\mathrm{CH}_{2}=\mathrm{CH}_{2}(80 \%)$ accompanied by minor $(20 \%)$ loss of ethyl radical (Scheme 3 ). This is also reflected in the composition of $\mathrm{Se}_{2}$ and $\mathrm{SeS}$ ion clusters. For example, the $\mathrm{Se}_{2}$ cluster in MeSeSeMe consists exclusively of $\mathrm{Se}_{2}^{-+}$. Replacement of one methyl group by ethyl group (in MeSeSeEt) results in a cluster of $75 \% \mathrm{Se}_{2}^{++}$and $25 \%$ contribution of $\mathrm{HSeSe}^{+}$. Further replacement of the methyl group (EtSeSeEt) results in a cluster of about $60 \% \mathrm{HSeSeH}^{++}$and $20 \% \mathrm{HSeSe}^{+}$(besides $\mathrm{Se}_{2}^{++}$itself).

Detailed inspection of the $\mathrm{C}_{2} \mathrm{H}_{n}$ Se cluster composition in dimethyldiselenide, ethylmethyldiselenide, and diethyldiselenide shows that the $\mathrm{C}_{2} \mathrm{H}_{n}$ Se pattern, similar
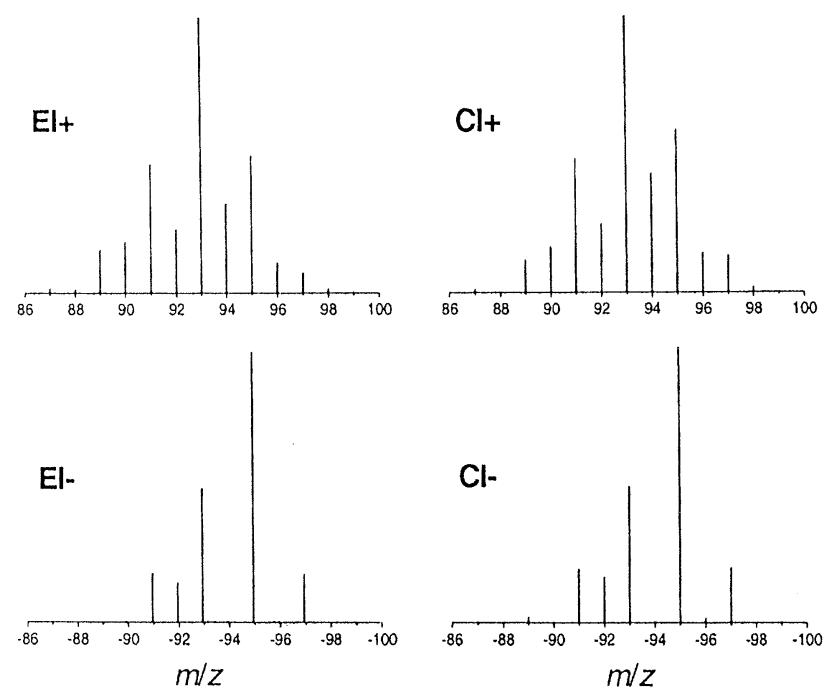

Figure 3. $\mathrm{CH}_{n} \mathrm{Se}$ cluster in mass spectra of dimethyldiselenide obtained under $\mathrm{EI}+, \mathrm{EI}-, \mathrm{CI}+$, and $\mathrm{CI}-$ ionization modes.

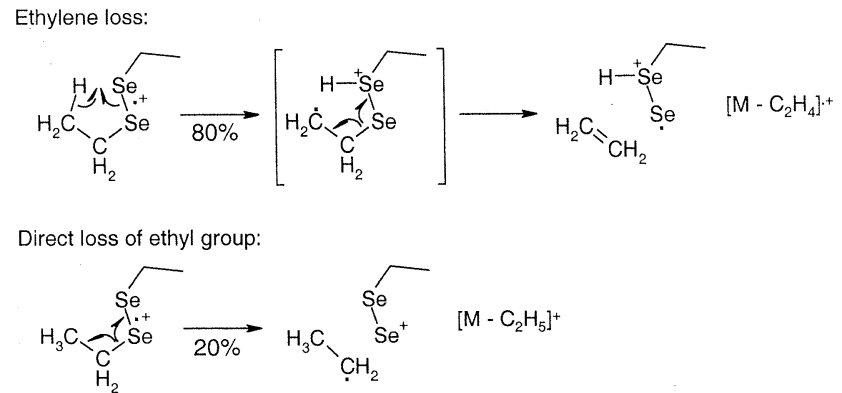

Scheme 3. Loss of ethyl group from diselenides in EI+ ionization mode.

to $\mathrm{CH}_{n} \mathrm{Se}$, consists of several species. In the presence of an ethyl group attached to a selenium atom (MeSeSeEt and EtSeSeEt), the $\mathrm{C}_{2} \mathrm{H}_{n}$ Se cluster consists of about 35\% $\mathrm{C}_{2} \mathrm{H}_{3} \mathrm{Se}, 20 \% \mathrm{C}_{2} \mathrm{H}_{4} \mathrm{Se}$, and $40 \% \mathrm{C}_{2} \mathrm{H}_{5} \mathrm{Se}$. On the other hand, isotope pattern deconvolution of $\mathrm{EI}+\mathrm{C}_{2} \mathrm{H}_{n} \mathrm{Se}$ pattern in MeSeSeMe (allowing for $n=0-6$ ) shows the prevailing presence $(>95 \%)$ of only $\mathrm{C}_{2} \mathrm{H}_{5}$ Se species, which is consistent with the selenoseleninyl rearrangement yielding $\mathrm{CH}_{2}=\mathrm{Se}-\mathrm{CH}_{3}^{+}$. MeSeSeEt and EtSeSeEt fragmentation, however, yields the species pattern consisting of $\mathrm{C}_{2} \mathrm{H}_{3} \mathrm{Se}, \mathrm{C}_{2} \mathrm{H}_{4} \mathrm{Se}$ and $\mathrm{C}_{2} \mathrm{H}_{5} \mathrm{Se}$ in the ratio of about 2:1:2.

Although EI+ is established as a standard ionization technique for structural confirmation, extensive fragmentation of aliphatic diselenides may lead to the loss of information regarding the internal bonding. For example, the methylseleno cluster (around $m / z=93-95$ ) is formed also from ethylseleno species and thus MeSeSEt and EtSeSMe are hard to distinguish from their EI+ spectra, as the positive charge is exclusively retained by Se-species due to the lower ionization energy of Se $(9.75$ $\mathrm{eV})$ compared to $\mathrm{S}(10.36 \mathrm{eV})$ and $\mathrm{C}(11.26 \mathrm{eV})$.

\section{EI- Ionization}

Negative electron impact ionization renders $\mathrm{M}^{-}$molecular ions. However, the inherent instability of the negatively charged diselenides and selenosulfenates is apparent in the EI- mass spectrum of dimethyldiselenide, where the molecular ion is no longer the most abundant (as in EI+). The reason behind this is that in negative ionization mode a valence electron is being added to the lowest unoccupied molecular orbital (LUMO) of the molecule. In aliphatic disulfides, diselenides and selenosulfenates the LUMO is a $\sigma_{\pi}(X-X)^{*}$ anti-bonding orbital [13] and thus the addition of an electron results in $\sigma_{\pi}(\mathrm{X}-\mathrm{X})^{*}$ anti-bonding orbital now being the HOMO (see Figure 4). This leads to a substantial increase of the $X-X$ bond length and eventual fragmentation along this bond favored by the repulsion of the $\sigma_{\pi}(\mathrm{Se}-\mathrm{Se})^{*}$ anti-bonding HOMO. In agreement with this, it is experimentally estimated that low energy electron capture in disulfides lowers the S-S bond dissociation energy by about $40 \%$ [14].

The Se-C and Se-Se bond energies in negatively 


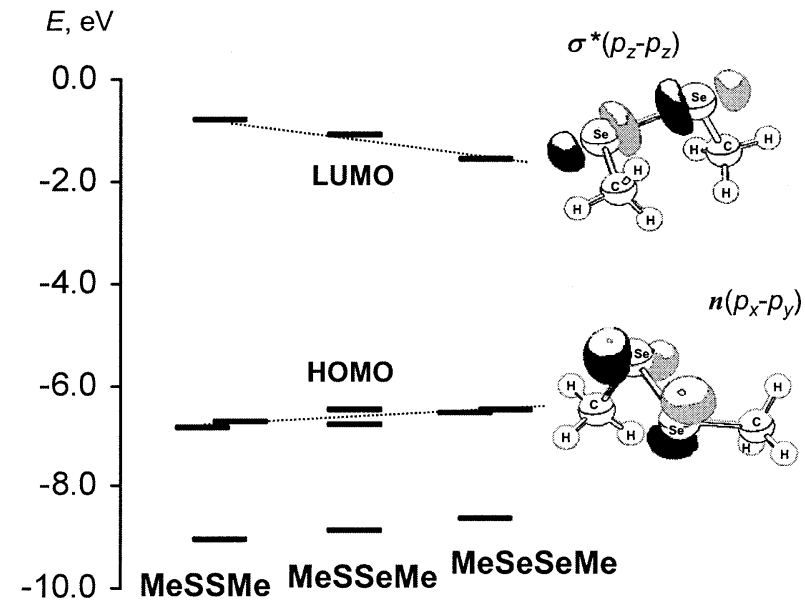

Figure 4. Highest occupied and lowest unoccupied molecular orbitals (HOMO and LUMO) in dimethyldisulfide, selenosulfenate and diselenide. Calculated using ub3lyp/6-311+G(2d).

charged dimethyldiselenide are very close (Table 1) and therefore the rupture of $\mathrm{Se}-\mathrm{C}$ and $\mathrm{Se}-\mathrm{Se}$ bonds is assumed to be comparably favorable, which is observed experimentally (see EI- and CI- in Figure 2).

Note that the secondary fragmentation in EI- occurs to a much lesser extent: The $\mathrm{CH}_{n} \mathrm{Se}$ cluster is entirely $\mathrm{CH}_{3} \mathrm{Se}^{-}$(Figure 3) and no selenoseleninyl rearrangement is observed (which would lead to the formation of $\mathrm{CH}_{2} \mathrm{SeCH}_{3}$ ). Also, elimination of the ethylgroup occurs only via the loss of ethyl radical, $\mathrm{CH}_{2} \mathrm{CH}_{3}$. The loss of ethyl radical in negative ionization (and not neutral ethylene loss as seen in EI+) is favored as it leaves a negatively charged even-electron selenium-containing anion.

It is interesting to note that the formation of various adducts has been observed for asymmetrical diselenides and selenosulfenates in EI- conditions (see Figure 5). Formation of symmetrical diethyldiselenide from the asymmetrical $\mathrm{CH}_{3} \mathrm{SSeCH}_{2} \mathrm{CH}_{3}$ in the EIconditions is evident. Similarly, formation of diethyldiselenide has been observed in the EI- mass spectra of ethylmethyldiselenide. Formation of these species might seem like a reverse chalcogen exchange reaction (toward symmetrical species) which occurs between the neutral species and the most abundant negative fragments, $\mathrm{RXSe}^{-}$. However, in this case a distorted Se isotope pattern might be expected in the EI- mass spectra of MeSeSeEt due to the overlap of the $\mathrm{EtSeSe}^{-}$ and $\mathrm{MeSeSeMe}{ }^{-}$, which is not observed. Similarly, this reaction cannot be explained by the interaction of the negatively charged molecular ion and its main fragmentation product, $\mathrm{CH}_{3} \mathrm{SSe}^{-}$. In such a case, formation of negatively charged dimethyldisulfide should be observed, which is not apparent.

A major difficulty in studying EI- fragmentation pathways lies in fact that this technique has about three orders of magnitude higher detection levels in comparison to EI+ and lower abundance ions thus are hard to measure with high confidence. However, regardless of

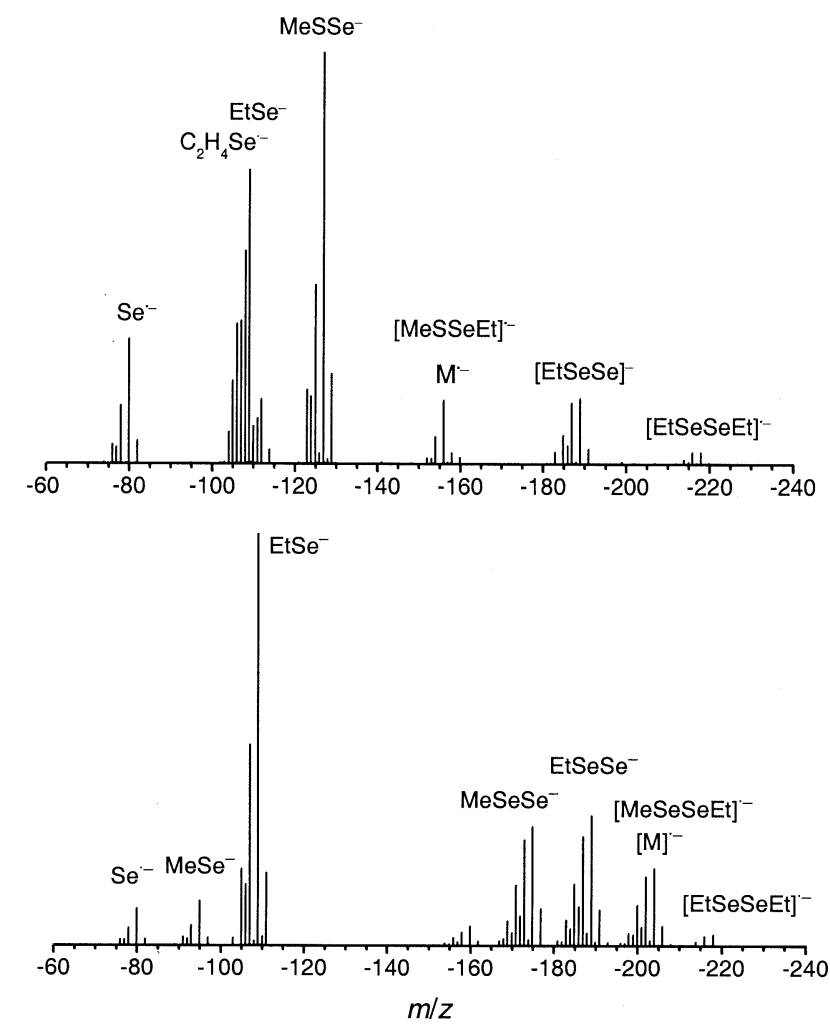

Figure 5. High resolution EI- mass spectra of ethylmethylselenosulfenate (EtSeSMe) and diselenide (MeSeSeEt) showing the formation of symmetric diselenides in negative ionization.

the mechanism, the formation of symmetrical chalcogen exchange reaction in EI- conditions has negative implications for molecular ion assignment. Due to this reaction, odd-electron symmetrical diselenides appear in the mass spectra at the masses larger than molecular ion (as shown in Figure 5). As a result, this might lead to erroneous molecular ion assignment taking into account the low intensity of molecular ion.

\section{CI+ Ionization}

In positive chemical ionization a formation of $[\mathrm{M}+\mathrm{H}]^{+}$ ions takes place and the ratio of $\mathrm{M}^{++}$to $[\mathrm{M}+\mathrm{H}]^{+}$under normal operating conditions (using isobutane) is close to 1:1. Very small amounts of reagent gas based adduct formation are observed when using isobutane, the main adducts being $\left[\mathrm{M}+\mathrm{C}_{3} \mathrm{H}_{5}\right]^{+}$and $\left[\mathrm{M}+\mathrm{C}_{4} \mathrm{H}_{9}\right]^{+}$. The relative abundance of these adducts with respect to the molecular ion cluster usually did not exceed 1-4\% for MeSeSeMe, MeSeSeEt and EtSeSeEt. As seen in Figure 6 , the loss of methyl group from the $\mathrm{M}^{++}$and $[\mathrm{M}+\mathrm{H}]^{+}$ ion cluster renders clear isotopic pattern of selenium, suggesting the presence of single species (supported also by isotope pattern superimposition calculations). This, however, is possible only by ${ }^{\circ} \mathrm{CH}_{3}$ radical loss from $\mathrm{M}^{+}$and neutral $\mathrm{CH}_{4}$ loss from the protonated [M+ $\mathrm{H}]^{+}$. Similar behavior, however, is not observed in the case of the ethyl group. Isotope cluster calculations of diethyldiselenide mass spectra suggest that protonated 

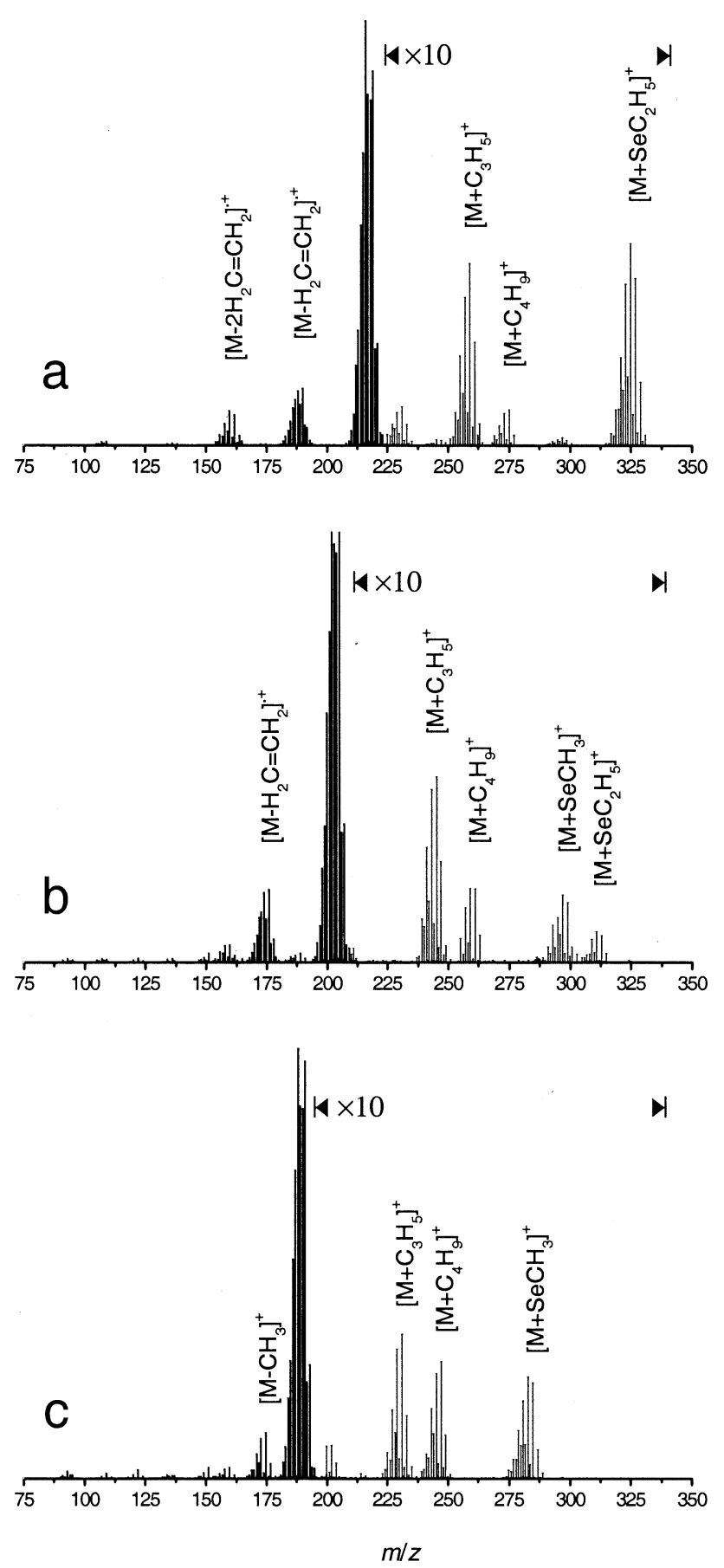

Figure 6. High resolution $\mathrm{CI}+$ mass spectra of EtSeSeEt (a), MeSeSeEt (b), and MeSeSeMe (c) showing the intermolecular association of the species.

diethyldiselenide loses ethylradical $\left(\mathrm{C}_{2} \mathrm{H}_{5}^{*}\right)$, ethane $\left(\mathrm{CH}_{3}-\mathrm{CH}_{3}\right)$, and ethene $\left(\mathrm{CH}_{2}=\mathrm{CH}_{2}\right)$ yielding clusters of $\mathrm{EtSeSeH}^{+}, \mathrm{EtSeSe}^{+}$, and $\mathrm{EtSeSeH}_{2}^{+}$.

Interestingly, formation of $[\mathrm{M}+\mathrm{RSe}]^{+}$adducts from diselenides and selenosulfenates is observed in the CI+ ionization process. This occurs as a minor process and the $[\mathrm{M}+\mathrm{RSe}]^{+}$ion abundances are usually about $1-5 \%$ relative to the $\mathrm{M}^{+}$. Similar adduct formation is observed also in the EI+ mass spectra, however, due to

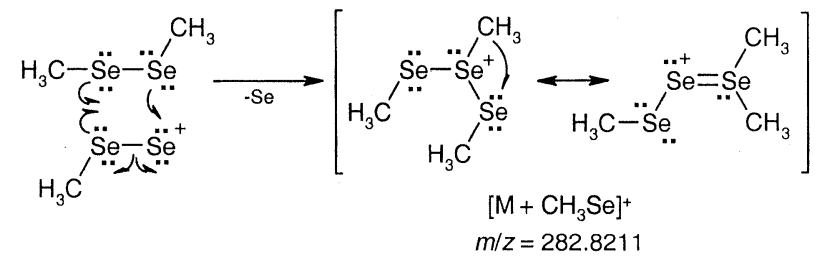

Scheme 4. Explanation of the formation of methyl selenium adducts from dimethyldiselenide in $\mathrm{EI}+$ and $\mathrm{CI}+$ conditions.

the extensive fragmentation processes, the abundance of $[\mathrm{M}+\mathrm{RSe}]^{+}$adducts in EI+ spectra is only about $0.1 \%$ relative to $\mathrm{M}^{+}$. The formation of $[\mathrm{M}+\mathrm{RSe}]^{+}$ adducts can be used for the structure confirmation studies of asymmetrical diselenides or selenosulfenates. For example, CI+ spectra of MeSeSEt exclusively show the formation of $\left[\mathrm{M}+\mathrm{CH}_{3} \mathrm{Se}\right]^{+}$adduct, and thus MeSeSEt can be easily distinguished from EtSeSMe from their chemical ionization spectra. No formation of $[\mathrm{M}+\mathrm{RS}]^{+}$adducts has been observed in selenosulfenates. If the molecular association occurs by interacting of the molecular species, such a process is suspected then to occur in the field ionization (FI+) experiments. In field ionization the valence electron tunnels through its confining boundaries in the presence of the strong applied electric field. This is a very soft ionization technique, which results in the formation of odd-electron molecular ion. No adduct formation (or fragmentation) from diselenides or selenosulfenates was observed in FI+ (data not shown) suggesting that the formation of the $[\mathrm{M}+\mathrm{RSe}]^{+}$adducts occurs by interaction of fragment ions (not present in FI+) with neutral molecular species as shown in Scheme 4.

This process is similar to the selenium exchange reaction, however the increased bond energies in the presence of the positive charge greatly favor molecular association rather than exchange.

\section{CI- Ionization}

Negative chemical ionization renders an almost exclusively intact pattern of the molecular ion, $\mathrm{M}^{--}$, which is beneficial for fast isotope pattern recognition. This is an advantage over $\mathrm{CI}+$, where the molecular ion cluster is superimposed with $[\mathrm{M}+\mathrm{H}]^{+}$isotopic pattern. This leads to the normal (Gaussian) isotope distribution pattern thereby losing the characteristic pattern of $\mathrm{Se}_{n}$ clusters and thus only high resolution MS instrumentation can confirm the number of selenium atoms in the molecular ion cluster when doing CI+. Similar to EI-, $\mathrm{CI}-$ equally favors the cleavage of Se-Se (and Se-S) in addition to the Se-C rupture (see Figure 2). However, fragmentation occurs to a much lesser extent. Although CI- shows intense molecular ions for dichalcogenides, it should be used with care because of the existing possibility of fragmentation along the chalcogen-chalcogen bond molecular ion might be absent in lesser stable species, such as trichalcogenides (data not shown). 


\section{Conclusions}

Mass spectral study of aliphatic diselenides shows that the principles of physical and organic chemistry are applicable to the fragmentation and rearrangement of ions in the gas phase. In general, positive ionization techniques favor the rupture of the C-Se bond, while negative ionization favors the rupture along the Se-Se bond. This principle is observed with $\mathrm{EI}+, \mathrm{CI}+, \mathrm{EI}-$, and CI- ionization modes. In addition, mass spectral characterization of diselenides and selenosulfenates can be achieved not only by detailed inspection of the molecular fragmentation (for example in $\mathrm{EI}+$ or $\mathrm{CI}-$ ) but also by inspecting the pathways of molecular association, as observed in $\mathrm{CI}+$ and EI- ionization conditions. Due to the inherent stability of the positively charged radicals of diselenides and selenosulfenates, $\mathrm{EI}+$ along with $\mathrm{CI}+$ are safe choices for obtaining molecular weight information. Although electron impact ionization gives intense molecular ion signals, extensive ion fragmentation and ion rearrangements can inhibit the conclusive identification in more complicated species. As a result of the different fragmentation pathways of positively and negatively charged diselenides or selenosulfenates, the complementary use of positive and negative ionization techniques is recommended for characterization of diselenides and related species.

\section{Acknowledgments}

The authors thank the Mass Spectrometry Facility at the University of Cincinnati for the use of GC-TOF-MS. Computing time was generously provided by the Molecular Modeling Services at the University of Cincinnati. JM thanks Anping Liu for his generous assistance in technical aspects of computational processing. This research was partially supported by NIEHS-SBRP grant ES04908 (JAC) and NSF grant CHE-0112322 (TLB).

\section{References}

1. Montes-Bayón, M.; Grant, T. D.; Meija, J.; Caruso, J. A. Selenium in Plants by Mass Spectrometric Techniques: Further Developments in Bioanalytical Methods. J. Anal. At. Spectrom. 2002, 17, 1015-1023.

2. Terry, N.; Zayed, A. M.; de Souza, M. P.; Tarun, A. S. Selenium in Higher Plants. Annu. Rev. Plant Physiol. Plant Biol. 2000, 51, 401-432.
3. Patching, S. P.; Gardiner, P. H. E. Recent Developments in Selenium Metabolism and Chemical Speciation: A Review. J. Trace Elements Med. Biol. 1999, 13, 193-214.

4. Rebane, E. Mass Spectrometric Studies on Organic SeleniumOxygen Compounds. 5: Mass Spectra of Simple Aliphatic and Aromatic Selenones. Chem. Scr. 1974, 6, 65-73.

5. Rebane, E. Mass Spectrometric Studies on Organic SeleniumOxygen Compounds. 4: Mass Spectra of Methaneseleninic Acid and Some Aromatic Seleninic Acids and Derivates. Chem. Scr. 1974, 5, 5-8.

6. Rebane, E. Mass Spectrometric Studies on Organic SeleniumOxygen Compounds. 3: Mass Spectra of Some Aliphatic and Aromatic Selenoxides. Chem. Scr. 1974, 7, 219-230.

7. Prabhakar, S.; Krishna, P.; Kundu, A.; Roy, S.; Vairamani, M. Mass Spectral Study of Substituted Allyl Aryl and Allyl Alkyl Selenides and Some Analogous Sulfides. Rapid Commun. Mass Spectrom. 1999, 13, 1564-1572.

8. Meija, J.; Bryson, J. M.; Vonderheide, A. P.; Montes-Bayón, M.; Caruso, J. A. Studies of Selenium Containing Volatiles in Roasted Coffee. J. Agric. Food Chem. 2003, 51, 5116-5122.

9. Frisch, M. J.; Trucks, G. W.; Schlegel, H. B.; Scuseria, G. E.; Robb, M. A.; Cheeseman, J. R.; Zakrzewski, V. G.; Montgomery, J. A. J.; Stratmann, R. E.; Burant, J. C.; Dapprich, S.; Millam, J. M.; Daniels, A. D.; Kudin, K. N.; Strain, M. C.; Farkas, O.; Tomasi, J.; Barone, V.; Cossi, M.; Cammi, R.; Mennucci, B.; Pomelli, C.; Adamo, C.; Clifford, S.; Ochterski, J.; Petersson, G. A.; Ayala, P. Y.; Cui, Q.; Morokuma, K.; Malick, D. K.; Rabuck, A. D.; Raghavachari, K.; Foresman, J. B.; Cioslowski, J.; Ortiz, J. V.; Baboul, A. G.; Stefanov, B. B.; Liu, G.; Liashenko, A.; Piskorz, P.; Komaromi, I.; Gomperts, R.; Martin, R. L.; Fox, D. J.; Keith, T.; Al-Laham, M. A.; Peng, C. Y.; Nanayakkara, A.; Gonzalez, C.; Challacombe, M.; Gill, P. M. W.; Johnson, B.; Chen, W.; Wong, M. W.; Andres, J. L.; Head-Gordon, M.; Replogle, E. S.; Pople, J. A. Gaussian 98; Gaussian, Inc.: Pittsburgh, PA, 1998.

10. Glendening, E. D.; Reed, A. E.; Carpenter, J. E.; Weinhold, F. NBO Version 3.1.

11. Meija, J.; Caruso, J. A. Deconvolution of Isobaric Interferences in Mass Spectra. J. Am. Soc. Mass Spectrom. 2004, 15, 654-658.

12. Tsai, J. H.; Hiserodt, R. D.; Ho, C.-T.; Hartman, T. G.; Rosen, R. T. Determination of Volatile Organic Selenium Compounds from the Maillard Reaction in a Selenomethionine-Glucose Model System. J. Agric. Food Chem. 1998, 46, 2541-2545.

13. Laitinen, R.; Pakkanen, T. Theoretical Investigation of the Sulfur-Selenium Bond. J. Mol. Struct. (Theochem) 1983, 91, 337-352.

14. Carles, S.; Lecomte, F.; Schermann, J. P.; Desfrançois, C.; Xu, S.; Nilles, J. M.; Bowen, K. H.; Berges, J.; Houee-Levin, C. Nondissociative Electron Capture by Disulfide Bonds. J. Phys. Chem. A 2001, 105, 5622-5626.

15. Tel'noi, V. I.; Sheiman, M. S. Thermodynamics of Organoselenium and Organotellurium Compounds. Uspheki Khimii 1995, 64, 330-337. 